\title{
Prevention of Wogonin on Colorectal Cancer Tumorigenesis by Regulating p53 Nuclear Translocation
}

\author{
Qian Feng ${ }^{1,2 t}$, Haojia Wang ${ }^{11}$, Jiaying Pang ${ }^{1}$, Liyan Ji', Jiada Han', Ying Wang', \\ Xiaoxiao Qi', Zhongqiu Liu' ${ }^{1 *}$ and Linlin Lu ${ }^{1 *}$ \\ ${ }^{1}$ International Institute for Translational Chinese Medicine, Guangzhou University of Chinese Medicine, Guangzhou, China, \\ ${ }^{2}$ The Postdoctoral Research Station, Guangzhou University of Chinese Medicine, Guangzhou, China
}

\section{OPEN ACCESS}

Edited by:

Vincent Kam Wai Wong, Macau University of Science and Technology, Macau

Reviewed by:

Jianxin Chen,

Beijing University of Chinese

Medicine, China

Songxiao $X u$,

Artron BioResearch, Inc., Canada

*Correspondence:

Zhongqiu Liu

liuzq@gzucm.edu.cn

Linlin Lu

Illu@gzucm.edu.cn

tThese authors have contributed equally to this work

Specialty section:

This article was submitted to Ethnopharmacology, a section of the journal

Frontiers in Pharmacology

Received: 17 September 2018 Accepted: 05 November 2018 Published: 23 November 2018

Citation:

Feng $Q$, Wang $H$, Pang J, Ji L, Han J, Wang Y, Qi X, Liu Z and Lu L (2018) Prevention of Wogonin on Colorectal Cancer Tumorigenesis by Regulating p53 Nuclear Translocation. Front. Pharmacol. 9:1356. doi: 10.3389/fphar.2018.01356
The tumor suppressor protein p53 plays an important role in the development and progression of colon cancer, and the subcellular organelle localization directly affects its function. Wogonin (5,7-dihydroxy-8-methoxyflavone), a mono-flavonoid extracted from root of Scutellaria baicalensis Georgi, possesses acceptable toxicity and has been used in colorectal cancer $(\mathrm{CRC})$ chemoprevention in pre-clinical trials by oncologist. However, the underlying anti-colon cancer mechanisms of wogonin are not yet fully understood. In the present study, the effect of wogonin on the initiation and development of colitis-associated cancer through p53 nuclear translocation was explored. AOM-DSS CRC animal model and human CRC HCT-116 cell model were used to evaluate the in vivo and in vitro anti-colon cancer action of wogonin. We observed that wogonin showed a dramaticlly preventive effect on colon cancer. Our results showed that wogonin caused apoptotic cell death in human CRC HCT-116 cell through increased endoplasmic reticulum (ER) stress. Meanwhile, excessive ER stress facilitated the cytoplasmic localization of p53 through increasing phosphor-p53 at S315 and S376 sites, induced caspase-dependent apoptosis and inhibited autophagy. Furthermore, we verified the chemoprevention effect and toxicity of wogonin in vivo by utilizing an AOM-DSS colon cancer animal model. We found that wogonin not only reduced tumor multiplicity, preserved colon length to normal $(6.79 \pm 0.34$ to $7.41 \pm 0.56, P<0.05)$ but also didn't induce side effects on various organs. In conclusion, these results explain the anti-tumor effect of wogonin in CRC and suggest wogonin as a potential therapeutic candidate for the therapeutic strategy in CRC treatment.

Keywords: wogonin, colorectal cancer, p53 nuclear translocation, endoplasmic reticulum stress, apoptosis, autophagy

\section{INTRODUCTION}

Colorectal cancer is the third most commonly prevalent malignancy worldwide and the fourth cause of cancer-related death (Das et al., 2016). More than 1 million new cases of CRC are reported annually and the incidence rate has been increasing. In 2012, nearly $10 \%$ of the total worldwide cancers, 1,361,000 new cases were reported and nearly half died from CRC (Siegel et al., 2017).

Abbreviations: AOM, azoxymethane; CHOP, C/EBP homologous protein; CRC, colorectal cancer; DSS, dextran sodium sulfate; ER, endoplasmic reticulum; IRE1 $\alpha$, inositol requiring enzyme 1 $\alpha$; PDI, protein disulfide isomerase; TUDCA, tauroursodeoxycholic acid. 
Although, the 5-year relative survival rate of CRC is on the rise, but the total death toll is still very high. Radiotherapy and chemotherapy are still preferred ways for the treatment of colon cancer. 5-fluorouracil (5-FU), leucovorin, oxaliplatin (FOLFOX) are conventional chemotherapy regimens, often fail to eradicate advanced CRC (Neska et al., 2016). Therefore, it will be a promising approach for cancer prevention and improve overall survival by utilizing food-derived, plant-derived, or pharmaceutical interventions with broad effectiveness and tolerable side effects to inhibit tumorigenesis and development.

The ER is a vital organelle and plays multifunctional roles in cellular signaling, stress response and cancer development present. Disruption of ER homeostasis is crucial for the development and maintenance of many cancers including breast (Lee et al., 2006), hepatocellular (Shuda et al., 2003), malignantgliomas (Pyrko et al., 2007), myeloma (Carrasco et al., 2007), and so on. Misfolded or unfolded proteins accumulation in the ER lumen induce ER stress, severe and prolonged ER stress has been confirmed could induce programmed cell death (Tabas and Ron, 2011; Yin et al., 2012). Apoptosis and autophagy are both forms of programmed cell death and autophagy is considered as a double-edged sword (Ouyang et al., 2012; Yu et al., 2017). It also has been reported that blockage of autophagy leads to aggravated ER stress and cell death (Burada et al., 2015; Sakitani et al., 2015).

A number of studies have shown that p53, a wellcharacterized tumor suppressor, gains or loses its activity depending on its localization (Iwao and Shidoji, 2014). There are several mechanisms that regulate the localization of p53 to different subcellular compartments. For instance, DNA damage and hypoxia always lead to the localization of p53 in the nucleus (Zhang et al., 2016), while ER stress and genotoxic stress could enhance cytoplasmic relocation of p53 (Qu et al., 2004). ER stress could facilitate the cytoplasmic localization of p53 through mediate directly or indirectly the phosphorylation of p53 at serine 315 or serine 376 , respectively (Shi and $\mathrm{Gu}, 2012$ ). The localization of p53 in cell could regulate the fate of cells. Through transactivation with its target genes, nuclear p53 could promote autophagy (Cheong et al., 2012). However, cytoplasmic p53 mainly inhibits autophagy through extranuclear transcription independent mechanisms (Tang et al., 2015). What's more, Jonathan Lévy indicated that the patients who are at high risk of developing CRC could suppress cancer development and growth through the inhibition of autophagy (Levy and Romagnolo, 2016).

Wogonin, a naturally mono-flavonoid, is extracted from root of Scutellaria baicalensis Georgi with various activities including anti-inflammatory, anti-tumor, and anti-microbial potentials. It has been reported that wogonin could improve p53 expression in hepatoma, glioblastoma, lung and colon cancer cells (Gao et al., 2011; Kim et al., 2012; Lee et al., 2012; Qian et al., 2014). Moreover, wogonin could modulate ER stress and eventually led to autophagy and/or apoptosis. But these roles were celltype and context dependent. For example, wogonin induced ER stress in IRE1 $\alpha$-dependent way in neuroblastoma, leukemia, and glioma cell lines (Tsai et al., 2012; Ge et al., 2015; Hu et al.,
2015). Conversely, wogonin could reverse ER stress induced by other stressors such as wogonin inhibited tunicamycininduced ER stress to protect dorsal root ganglion neuronsin rat (Xu et al., 2015). Therefore, it is necessary to further clarify whether the effect of wogonin on ER stress and p53 nuclear translocation to affect carcinogenesis and development of CRC.

In this study, the anti-tumor efficacy of wogonin was addressed in vivo by utilizing an AOM-DSS CRC animal model. The intrinsic mechanism of wogonin prevents CRC through production of ER-stress and p53 nuclear translocation was explored in vitro by using human CRC cell lines. Considering the important role of the intracellular localization of p53 in the development and progression of colon cancer, determining the effect of wogonin on $\mathrm{p} 53$ nuclear translocation may provide a new thought and method for the prevention on CRC tumorigenesis.

\section{MATERIALS AND METHODS}

\section{Cell Lines and Cell Culture}

Human colorectal carcinoma cells HCT-116 were obtained from the American Type Culture Collection (ATCC, Manassas, VA, United States). Cell culture reagents were purchased from Invitrogen (Gibco, Waltham, MA, United States). The cells were cultured in Dulbecco's modified eagle medium (DMEM) supplemented with 10\% (v/v) fetal bovine serum (FBS), $100 \mathrm{U} / \mathrm{mL}$ penicillin and $100 \mu \mathrm{g} / \mathrm{mL}$ streptomycin. All cells were maintained at $37^{\circ} \mathrm{C}$ in a humidified atmosphere of $5 \% \mathrm{CO}_{2}$.

\section{Chemicals, Reagents, and Antibodies}

Wogonin (purity $\geq 98 \%$, HPLC grade, confirmed by LC/MS/MS) was purchased from Dalian Meilun Biotech, Co., Ltd. (Dalian, China). All other chemicals were purchased from Sigma-Aldrich (St. Louis, MO, United States). Primary antibodies for LC3 I/II, Cleaved caspase 3, Cleaved caspase 9, IRE-1a, CHOP, PDI, Calnexin and secondary antibody Anti-rabbit IgG, HRPlinked were brought from Cell Signaling Technology, Inc. (Danvers, MA, United States). Primary antibody for p-p53(s15), p-p53(s376) and p-p53(s315) were purchased from Abcam, Inc. (Cambridge, MA, United States). Primary antibodies for GAPDH and Histone $\mathrm{H} 3$ and secondary antibody horseradish peroxidaselinked anti-mouse immunoglobulin $G$ were purchased from Santa Cruz Biotechnology, Inc. (Santa Cruz, CA, United States). Western blot detection reagents were obtained from Bio-Rad Laboratories (Hercules, CA, United States).

\section{MTT Cell Viability Assay}

The cell viability was measured via MTT assay. $2 \times 10^{3}$ cells/well were seeded into 96-well plates and cultured with different doses $(1 \sim 100 \mu \mathrm{M})$ of wogonin for $72 \mathrm{~h}$. At the end of treatment, $100 \mu \mathrm{L}$ of MTT $(0.5 \mathrm{mg} / \mathrm{mL})$ was added to each sample and incubated for $4 \mathrm{~h}$ at $37^{\circ} \mathrm{C}$. Then, the supernatants were discarded and $150 \mu \mathrm{L}$ of DMSO was added to dissolve the formazan crystals. Then optical density was determined using a microplate reader Victor X3 at 490 nm (PerkinElmer, Waltham, MA, United States). 


\section{Colony-Formation Assay}

HCT-116 cells (300 cells/well) were seeded in 6-well plates 1 day before treatment. DMEM $(2 \mathrm{ml})$ containing 10\% FBS was added to each well with a vehicle $(0.01 \%$ DMSO) or different concentration of wogonin ( 5 and $10 \mu \mathrm{M})$. After 2 weeks cultured, colonies with more than 50 cells were counted, the percentage of colony-forming rate was calculated to evaluate colony formation efficiency. Colony-formation rate was calculated as: (Colony counts experiment group/ Colony counts medium control group) $\times 100 \%$.

\section{Apoptosis Assay}

HCT-116 cells were seeded in 6 -well plates $\left(3 \times 10^{5}\right.$ cells/well $)$ 1 day before treatment. Then cells were treated with or without wogonin $(5$ and $10 \mu \mathrm{M})$ for $48 \mathrm{~h}$. The cells were harvested and processed for cell apoptosis analysis by using FITC Annexin V Apoptosis Detection Kit (BD Biosciences). Cell apoptosis was determined by using FACS Aria III (BD Biosciences) equipped with FlowJo v7.6. Annexin-V positive PI negative $\left(\mathrm{AV}^{+} \mathrm{PI}^{-}\right)$ signals represent cells in early apoptosis, while Annexin- $\mathrm{V}$ positive $\mathrm{PI}$ positive $\left(\mathrm{AV}^{+} \mathrm{PI}^{+}\right)$signals indicate cells in late apoptosis.

\section{Western Blot Analysis}

Cells were seeded in six-well plates with density of $4 \times 10^{5}$ cells/well, and treated with a vehicle $(0.01 \%$ DMSO) or 5 and $10 \mu \mathrm{M}$ wogonin for $72 \mathrm{~h}$. Cell lysates for protein blot analysis were prepared using standard RIPA buffer (Santa Cruz Biotechnology). Western blot analysis was performed to detect the expression levels of target proteins according to the literature (Martínez-Revelles et al., 2016). Briefly, $30 \mu \mathrm{g}$ protein was loaded on (10\%) SDS-PAGE and separated. After transmembrane, PVDF membranes were stained with primary and secondary antibodies. GAPDH or Histone H3 served as a loading control. The relative intensities of the protein bands were scanned and quantified using Quantity One Program (Bio-Rad).

\section{Real-Time PCR Analysis}

After wogonin treatment, total RNA was isolated using TRIzol and synthesized to cDNA by using a reverse transcription kit (TaKaRa, Shiga, Japan). SYBR Green real-time PCR amplification and detection were then performed by using an ABI 7500 system (Applied Biosystems, Foster City, CA, United States). The following primers were used: DDIT3, forward 5'-AATGAACGGCTCAAGCAGGA-3' and reverse 5'-AGCCAC TTCTGGGAAAGGTG-3'; XBP1, forward $5^{\prime}$-AAGTTCTGCT TCTGTCGGGG-3' and reverse $5^{\prime}$-TGCACGTAGTCTGAG TGCTG-3'; GAPDH, forward 5'-GGTGTGAACCATGAG AAGTATGA-3' and reverse 5'-GAGTCCTTCCACGATAC CAAAG-3'. Relative gene expression levels were normalized to GAPDH expression. The relative mRNA expression levels were analyzed by $2^{-\Delta \Delta \mathrm{Ct}}$.

\section{Immunofluorescence Assay}

The immunofluorescence assays in HCT-116 cells were seeded on $15 \mathrm{~mm}$ confocal dish with 5 or $10 \mu \mathrm{M}$ wogonin treating for
$48 \mathrm{~h}$ and fixed in cold 4\% paraformaldehyde. The confocal dishes were incubated with p-p53(ser15) (Abcam, 1:200). Detection of the primary antibody was performed using 1:200 Alexa Fluor ${ }^{\circledR}$ conjugated secondary antibody. The nucleus was stained with $0.1 \%$ DAPI and microfilament was stained with $0.1 \%$ phalloidin. The images were captured and analyzed using Leica TCS SP8 confocal microscope.

\section{Animal Model of AOM/DSS-Induced Colorectal Cancer}

All animals used in this study were obtained from Laboratory Animal Center of Sun Yat-sen University (Guangzhou, China) and approved by Animal Care and Use Committee (IACUC) in Guangzhou University of Chinese Medicine. Colorectal cancer was induced in 4-week-old male C57BL/6 mice via the intraperitoneal injection of AOM $(10 \mathrm{mg} / \mathrm{kg})$ while the mice were maintained with a regular diet and drinking water for 14 days and then mice were subjected with three cycles of DSS treatment, with each cycle consisting of the administration of $2 \%$ DSS for 7 days, followed by a 14-day recovery period with regular water. Mice were administered with 50 and $100 \mathrm{mg} / \mathrm{kg}$ wogonin daily from $5^{\text {th }}$ to $25^{\text {th }}$ week.

\section{Statistical Analysis}

All results are expressed as mean \pm standard deviation (SD) of values from three independent experiments. Data were statistical analyzed by one-way ANOVA using SPSS 18.0. $P$-values $<0.05\left(^{*}\right),<0.01\left(^{* *}\right),<0.001\left(^{* * *}\right)$ were considered as statistical significant between experiment and control groups.

\section{RESULTS}

\section{Wogonin Induced Cell Death in Human Colorectal Cancer Cells via Caspase-Dependent Apoptotic Pathway}

The anti-cancer effects of wogonin were first investigated in vitro in human colon cancer cells. Human colon cancer HCT116 cells were treated by wogonin at various concentrations (1$100 \mu \mathrm{M}$ ) for $72 \mathrm{~h}$ and analyzed by MTT assay. Result showed that wogonin exhibits a dose-dependent reduction in HCT116 cell viability (Figure 1A). After treatment, wogonin significantly inhibited cell growth with the maximum inhibition ratio of $68.67 \pm 2.80 \%$ at $100 \mu \mathrm{M}$ in HCT-116 cells (Figure 1A, $P<0.001)$. To further analyze the influence of wogonin in cell biology, wogonin at 5 and $10 \mu \mathrm{M}$, with respective cell inhibition of $29.47 \pm 3.34$ and $36.35 \pm 4.38 \%$, were chosen for following studies. Moreover, the anti-proliferation effects of wogonin in HCT-116 cells were evaluated by colony-formation assay. The result confirmed that wogonin inhibited HCT-116 cell productive capacity in a concentration-dependent manner (Figure 1B, $P<0.001$ ), the respective cell colony formation rate is $54.58 \pm 5.60$ and $26.78 \pm 1.55 \%$ after wogonin treatment at 5 and $10 \mu \mathrm{M}$ for 14 days. Collectively, our results suggest that 
A<smiles>COc1c(O)cc(O)c2c(=O)cc(-c3ccccc3)oc12</smiles>

B

HCT116

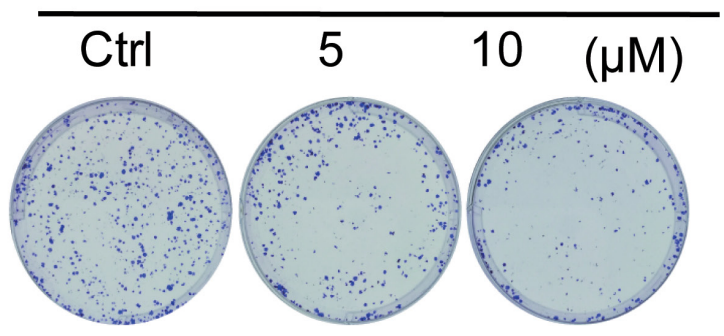

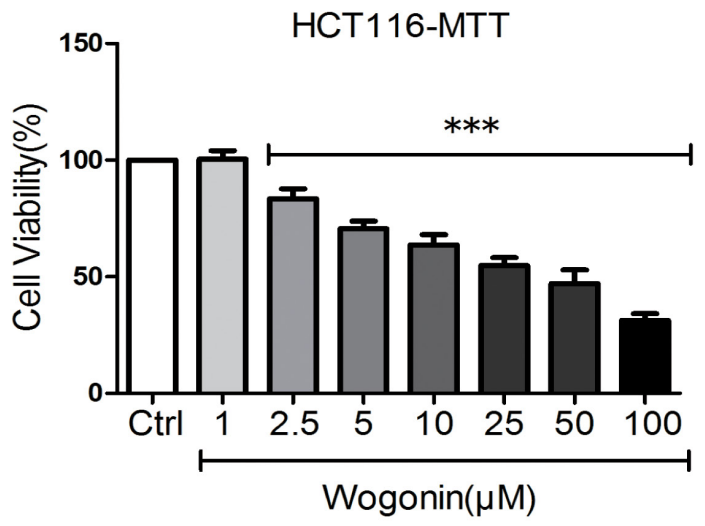

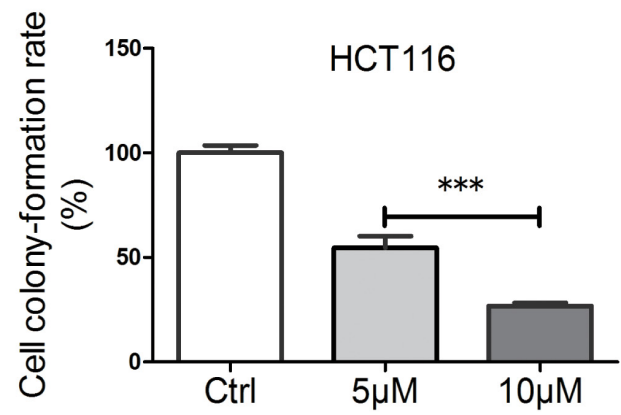

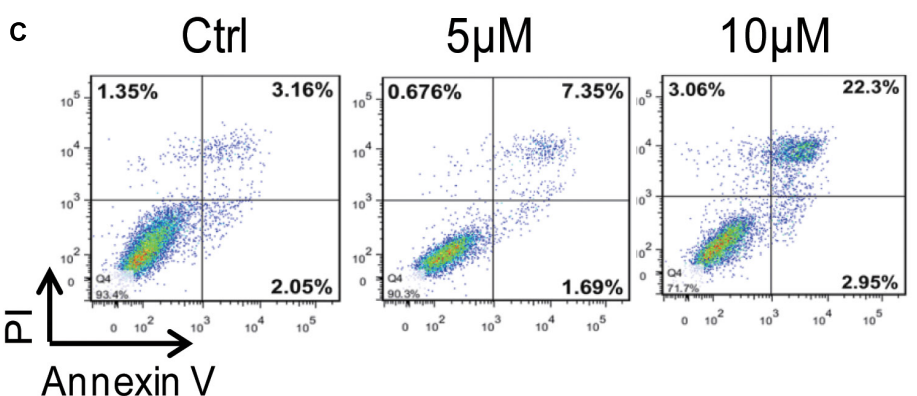

D

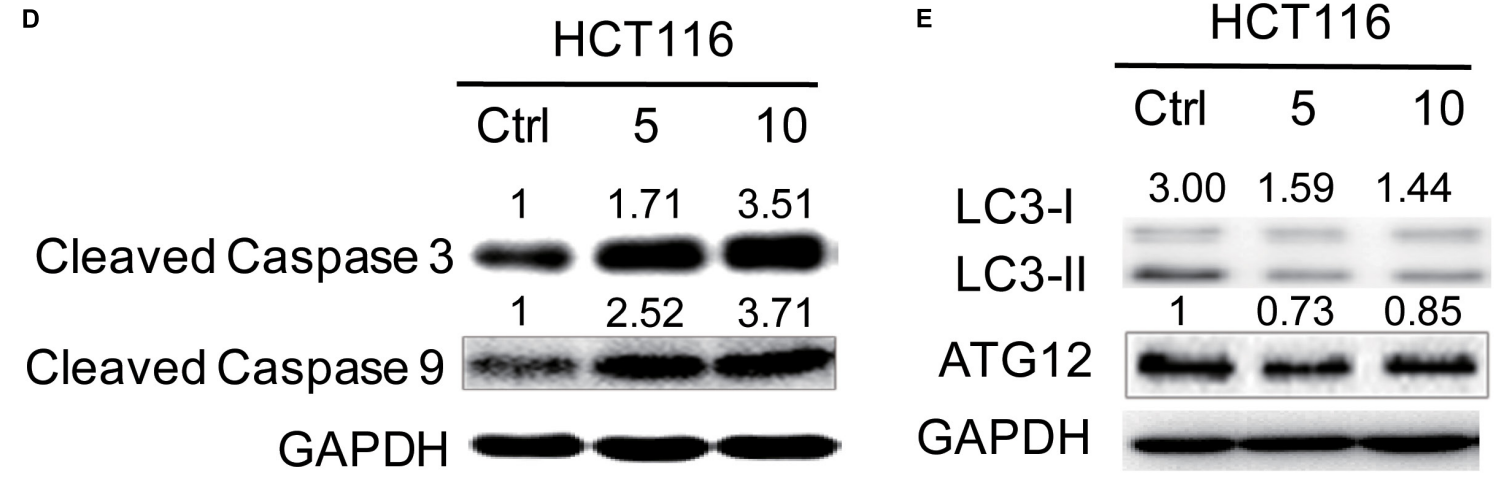

E

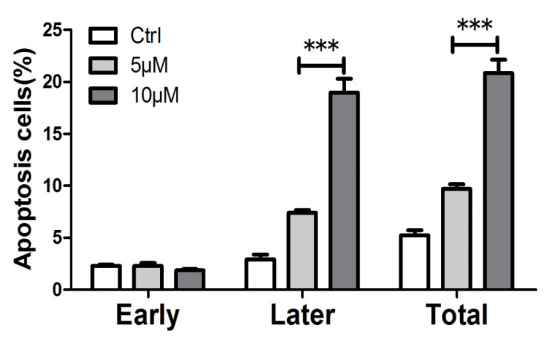

FIGURE 1 | Wogonin induced cell death via caspase-dependent apoptotic pathway in human CRC cells. (A) Wogonin inhibited human CRC HCT-116 cells viability of in a concentration dependent manner. HCT-116 cells were treated with various concentrations (0-100 $\mu \mathrm{M})$ of wogonin for $72 \mathrm{~h}$ as measured by MTT assay. (B) Effects of wogonin on the colony formation abilities of HCT-116 cells. Colony formation rate was showed in histogram. (C) Effects of wogonin on apoptotic cell death in HCT-116 cells. HCT-116 cells treated with wogonin for $48 \mathrm{~h}$, and the proportion of apoptotic cells was determined by flow cytometry using Annexin V/propidium iodide (PI) double staining. (D) The expression levels of cleaved caspase 3 and 9 in 9 in HCT-116 cells after wogonin treatment were measured using Western blot assay. GAPDH was used as an equal loading control. (E) Western blot analysis of autophagy-related proteins including LC3-II and LC3-I in HCT-116 cells after wogonin treatment for $72 \mathrm{~h}$. GAPDH was used as an equal loading control. The results represent the mean of three independent experiments. Values represent mean $\pm \mathrm{SD}$, significant difference versus control group, ${ }^{* * *} P<0.001$. 
wogonin inhibits the colon cancer HCT-116 cell viability and proliferation.

To determine whether the inhibition effects of wogonin in CRC were caused by apoptosis or autophagy, flow cytometry and western blot were chosen for further analyzed. Flow cytometry analysis of AV and PI positivity showed that wogonin treatment induced significantly apoptosis in HCT-116 cells after $48 \mathrm{~h}$ (Figure 1C, $P<0.001$ ). The percentages of late apoptotic cells were significantly increased to $7.40 \pm 0.26$ and $21.40 \pm 0.10 \%$ after wogonin (5 and $10 \mu \mathrm{M})$ treatment compared with $2.93 \pm 0.45 \%$ in control group. Meanwhile, in order to understand the pathway involved in wogonininduced apoptosis, expression of cleaved caspase 3 and cleaved caspase 9, central executors of intrinsic apoptotic cell death, were detected by western blot. Protein levels of cleaved caspase 3 and cleaved caspase 9 were significantly increased by $3.52 \pm 0.48$ (Figure $1 \mathrm{D}, P<0.01$ ) fold and $3.72 \pm 0.15$ (Figure 1D, $P<0.001$ ) fold respectively after wogonin $(10 \mu \mathrm{M})$ treatment for $72 \mathrm{~h}$ (Figure 1D). LC3-II to LC3I conversion, the reliable autophagy markers for monitoring autophagic cell death, also were detected by western blot. As shown in Figure 1E, we found that conversion ratio of LC3-II to LC3-I was decreased $0.49 \pm 0.01$ fold $(P<0.01)$ after $10 \mu \mathrm{M}$ wogonin treatment for $72 \mathrm{~h}$ in HCT-116. All these data together indicated that wogonin induce human CRC HCT-116 cell death via caspase-dependent apoptotic pathway.

\section{Wogonin Caused Apoptotic Cell Death Through Increased Endoplasmic Reticulum Stress in Colorectal Cancer Cells}

It has been reported that wogonin could modulate ER stress and eventually led to autophagy and/or apoptosis (Ge et al., 2015; Hu et al., 2015). To determine whether ER stress was associated with wogonin-induced cell death, ER chaperones (CALNEXIN, PDI), ER-associated key sensors (IRE1 $\alpha$ ), downstream transcription factor CHOP and regulated genes DDIT3 and XBP1 were detected by western blotting and qRT-PCR in HCT-116 cells. As shown in Figure 2A, in HCT-116 cells, wogonin increased protein expression of PDI, CALNEXIN, IRE- $1 \alpha$ and CHOP in a dose-dependent manner. Particularly, PDI and CALNEXIN were significantly increased by $1.76 \pm 0.04$ fold $(P<0.001)$ and $2.65 \pm 0.11$ fold $(P<0.001)$ after wogonin $(10 \mu \mathrm{M})$ treatment. Moreover, IRE- $1 \alpha$ showed $1.48 \pm 0.03$ fold increase $(P<0.01)$ after wogonin $(10 \mu \mathrm{M})$ treatment. Furthermore, CHOP was significantly increased by $2.03 \pm 0.16$ fold $(P<0.01)$ after treatment with $10 \mu \mathrm{M}$ wogonin for $72 \mathrm{~h}$ compared to control group. What's more, PCR results also confirmed that mRNA levels of ER stress associated downstream gene DDIT3 and XBP1 in HCT-116 cells were both up-regulated by $42.91 \pm 0.34$ fold (Figure 2B, $P<0.001$ ) and $8.10 \pm 0.79$ fold (Figure 2B, $P<0.001)$ after wogonin $(10 \mu \mathrm{M})$ treatment, respectively.

Tauroursodeoxycholic acid is an ambiphilic bile acid and a specific inhibitor of ER stress (Fan et al., 2017;
Fernandez-Bautista et al., 2017). Flow cytometry result showed that the apoptotic effect of wogonin $(10 \mu \mathrm{M})$ caused in HCT-116 cells was completely reversed by TUDCA (500 $\mu \mathrm{M})$ (Figure 2C). The percentages of apoptotic cells were significantly decreased from $25.36 \pm 0.06$ to $17.40 \pm 0.26 \%$ after wogonin $(10 \mu \mathrm{M})$ and TUDCA $(500 \mu \mathrm{M})$ treatment. Meanwhile, cleaved caspase 3 and cleaved caspase 9 protein expressions were revealed $0.61 \pm 0.02$ fold $(P<0.05)$ and $0.52 \pm 0.03$ fold $(P<0.01)$ decrease after treating with TUDCA $(500 \mu \mathrm{M})$ and wogonin $(10 \mu \mathrm{M})$ for $72 \mathrm{~h}$ in HCT-116 cells (Figure 2D). IRE-1 $\alpha$ and PDI were exhibited $0.57 \pm 0.03$ fold $(P<0.05)$ and $0.61 \pm 0.03$ fold $(P<0.05)$ decrease after treating with TUDCA $(500 \mu \mathrm{M})$ and wogonin $(10 \mu \mathrm{M})$. Besides that, the level of conversion ratio of LC3-II to LC3-I was increased by $3.46 \pm 0.16$ fold $(P<0.001)$ compare with wogonin $(10 \mu \mathrm{M})$ treat alone for $72 \mathrm{~h}$ in HCT-116 cells. All these results confirmed that wogonin caused apoptotic cell death in human CRC HCT-116 cell through increased ER stress.

\section{Wogonin-Induced Endoplasmic Reticulum Stress Modulated Nuclear Translocation of p-p53}

Given that ER stress was regulated by wogonin, this pathway could converge into transcription factor p53 by controlling its nucleocytoplamic shuffling (Qu et al., 2004; Shi and Gu, 2012). To investigate how p-p53 shuffle was affected by wogonin, we studied the cytoplasm/nucleus translocation of p-p53 by western blot and immunofluorescence. For total protein, after wogonin $(10 \mu \mathrm{M})$ treatment for $72 \mathrm{~h}$, phosphorylation at serine 315 and serine 376 of p53 both were increased by $1.98 \pm 0.09$ fold $(P<0.01)$ and $3.78 \pm 0.12$ fold $(P<0.001)$ in HCT-116 cells (Figure 3A). To further clear the distribution of $\mathrm{p}$-p53, we separated the nuclear and cytoplasmic proteins to evaluate the localization of p-p53. In HCT-116 cells cytoplasm, wogonin treatment exhibits a dosedependent protein expressions increase of phosphorylation at serine S376 of p53. Wogonin treatment at $10 \mu \mathrm{M}$ exhibited $3.72 \pm 0.06$ fold increase $(P<0.001)$ compare to control group. While in nucleus, expressions of S376 of p53 were decreased to $0.51 \pm 0.02(P<0.05)$ fold and $0.47 \pm 0.04$ fold $(P<0.01)$ after wogonin $(5$ and $10 \mu \mathrm{M})$ treatment (Figure 3A).

Immunofluorescence assay was used to further confirm whether wogonin could influence the cytoplasmic/nuclear distribution of phosphorylation of p53. In HCT-116 cells, phosphorylation at serine 15 of p53 in nucleus was dramatically decreased, and the ratio of phosphorylation at serine 15 of p53 in nucleus/cytoplasm was decreased to $0.47 \pm 0.002$ fold $(P<0.001)$ after wogonin treatment for $48 \mathrm{~h}$ (Figure 3B). At the same time, ER stress inhibitor, TUDCA $(500 \mu \mathrm{M})$ completely reversed the decreased effect of the ratio of phosphorylationof p53 in nucleus/cytoplasm caused by wogonin $(10 \mu \mathrm{M})$ (Figure 3C). These findings suggested that wogonin regulated nuclear translocation of p-p53 by increasing the ER stress response to inhibit autophagy in HCT116 colon cancer cells. 

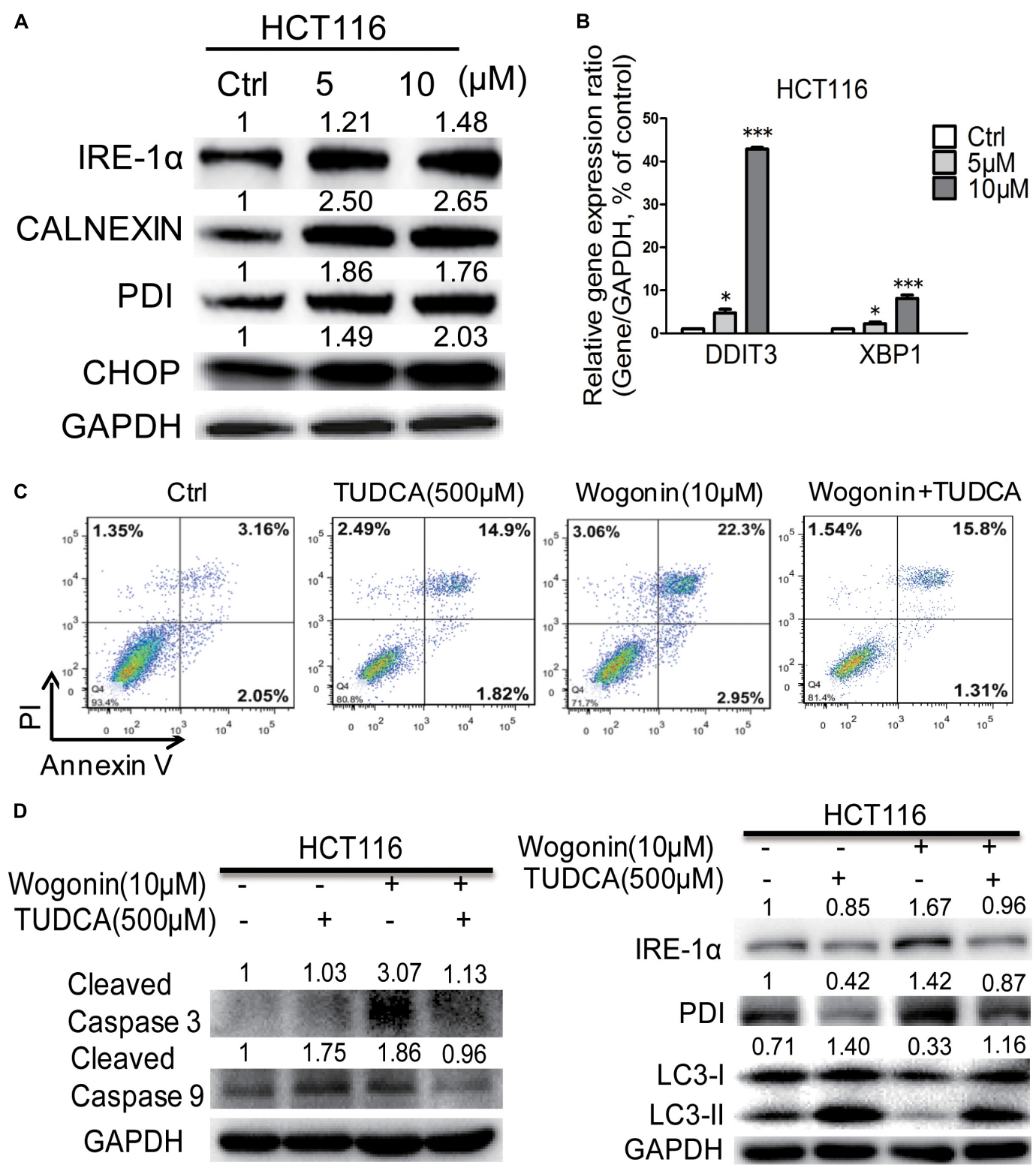

FIGURE 2 | Wogonin caused apoptotic cell death through increased ER stress in human CRC HCT-116 cell. (A) IRE-1 $\alpha$, CHOP, PDI, and CALNEXIN protein levels in HCT-116 cells treated with wogonin and vesicle (0.1\% DMSO) for $72 \mathrm{~h}$ were detected using western blot assay. The band intensities were quantified by Quantity One and represented as bar graph in the right panel. GAPDH was used as an internal control. (B) Gene expression of DDIT3 and XBP1 were determined by real-time PCR after treatments with indicated concentrations of wogonin for $72 \mathrm{~h}$. GAPDH served as housekeeping gene. (C) Apoptotic effects in HCT-116 cells after wogonin treatment $(10 \mu \mathrm{M})$ with/without TUDCA $(500 \mu \mathrm{M})$ for $48 \mathrm{~h}$, and the proportion of apoptotic cells was determined by flow cytometry using Annexin V/propidium iodide (PI) double staining. (D) Protein expression levels of cleaved caspase 3, cleaved caspase 9, IRE-1 $\alpha$, PDI, LC3-II to LC3-I in HCT-116 cells after wogonin treatment $(10 \mu \mathrm{M})$ with/without TUDCA $(500 \mu \mathrm{M})$ for $72 \mathrm{~h}$. GAPDH was used as an equal loading control. Experiments were performed in triplicate and data represent as mean $\pm \mathrm{SD}$, significant difference versus control group, ${ }^{*} P<0.05 ; * * * P<0.001$

\section{Wogonin Inhibited Tumorigenesis in AOM/DSS-Induced CRC Mice Model Through Increasing Endoplasmic Reticulum Stress}

The AOM-DSS colon cancer animal model was used to evaluate the chemopreventive effect and toxicity of wogonin in vivo. As shown in Figure 4A, mice developed tumors when they were given with $\mathrm{AOM}(10 \mathrm{mg} / \mathrm{kg})$ by i.p. injection at week 4 and fed with DSS (2\%) water for three-cycles. After consecutive 21 weeks of wogonin oral administration, there was no significant body weight loss in mice (Supplementary Figure 1A). At the termination of experiment, tumor node multiplicity was calculated as tumors stratifying by size according to diameter, tumor incidence is reduced from $80.00 \%$ in model group to 54.55 and $66.67 \%$ in 50 and 
A

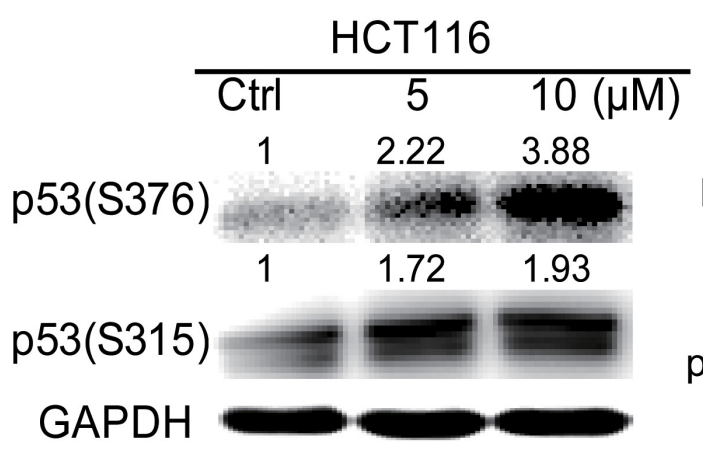

HCT116

$\begin{array}{llll}\text { Ctrl } 5 & 10 & (\mu \mathrm{M})\end{array}$

$\begin{array}{lll}1 & 3.28 & 3.72\end{array}$

p53(S376)

GAPDH

p53 (S376)

Histone $\mathrm{H} 3$
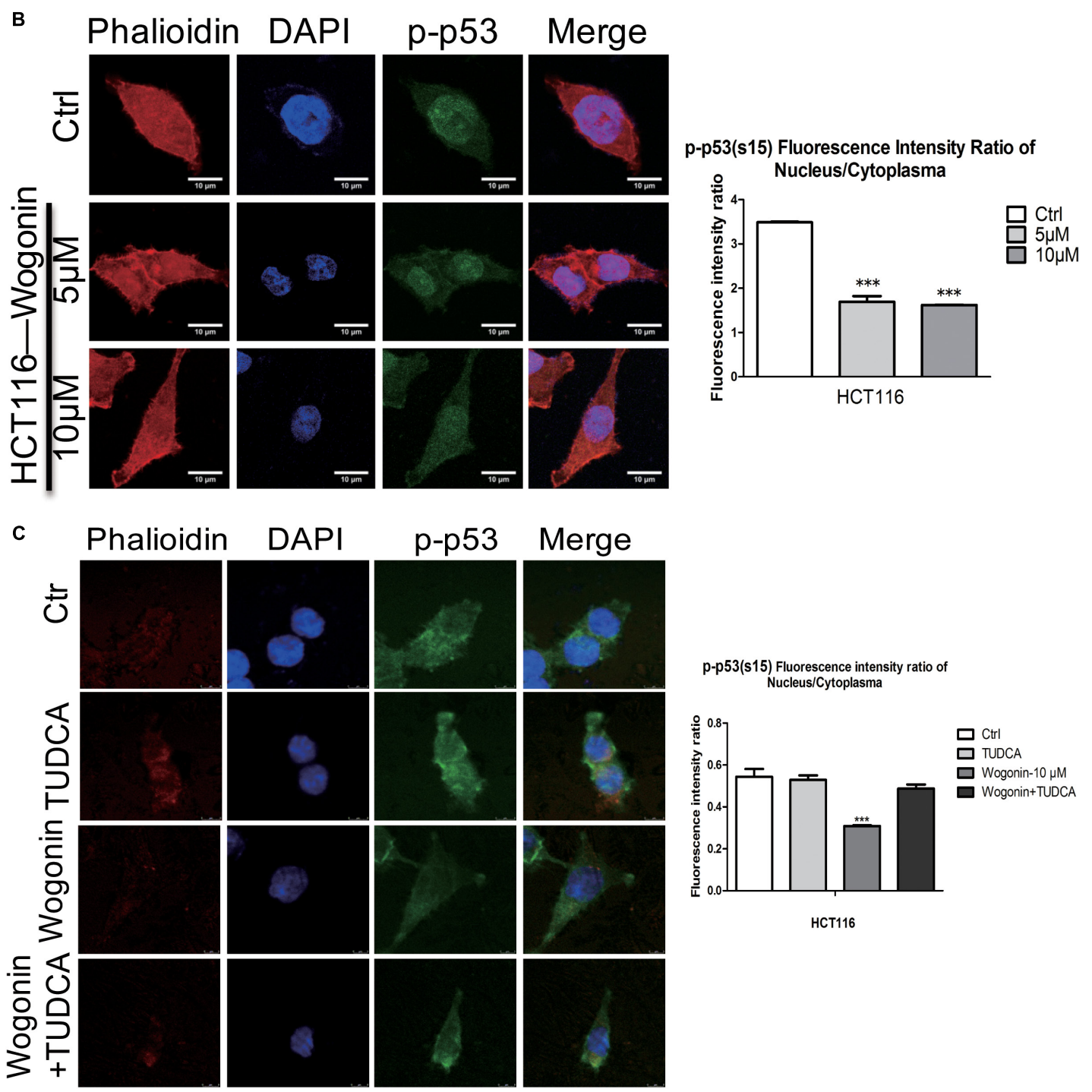

$p-p 53(s 15)$ Fluorescence intensity ratio of Nucleus/Cytoplasma

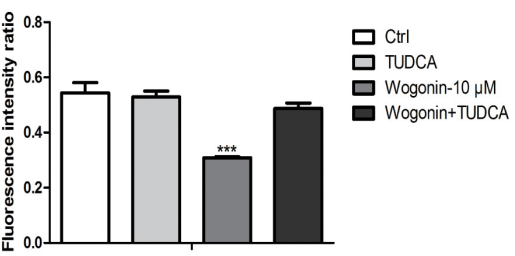

HCT116

FIGURE 3 | Wogonin modulated nuclear translocation of p-p53 through increased ER stress. (A) Total protein, cytoplasm protein and nuclear protein expressions of p53(ser315) andp53 (ser376) were examined by western blot after wogonin treated for $72 \mathrm{~h}$ in HCT-116 cells. Histone H3 or GAPDH was used as an internal control. (B) The distribution of p53(ser15) were visualized by immunofluorescence using p53(ser15) antibody (Green), nuclei were counterstained with DAPI (Blue) and cytomembrane were counterstained with phalloidine (Red). p53(ser15) fluorescence intensity ratio of cytoplasm/nucleus was expressed in histogram.

(C) Immunofluorescence assay was conducted to evaluate the ratio of phosphorylation at serine 15 of p53 in nucleus/cytoplasm. HCT116 cells were treated with wogonin $(10 \mu \mathrm{M})$, TUDCA $(500 \mu \mathrm{M})$, and wogonin combined with TUDCA for $48 \mathrm{~h}$ in HCT-116 cells. Values represent mean \pm SD, significant difference versus control group, ${ }^{* * *} P<0.001$. 
A

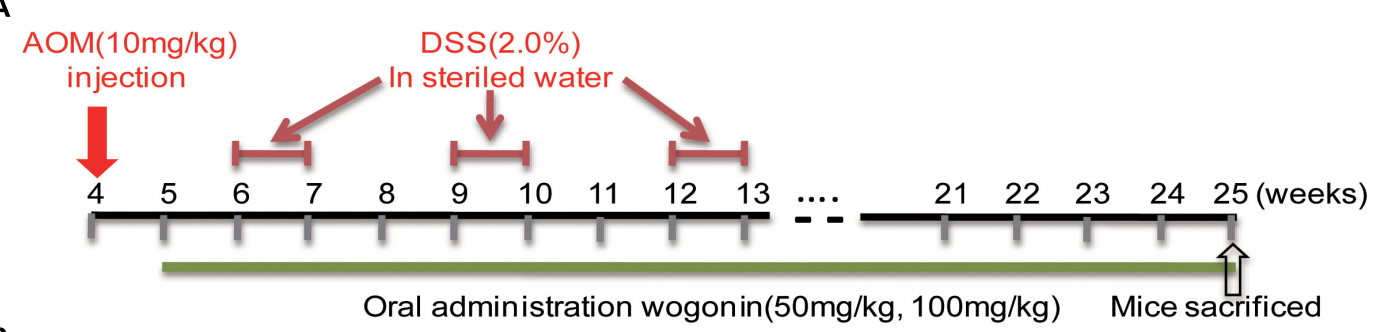

B
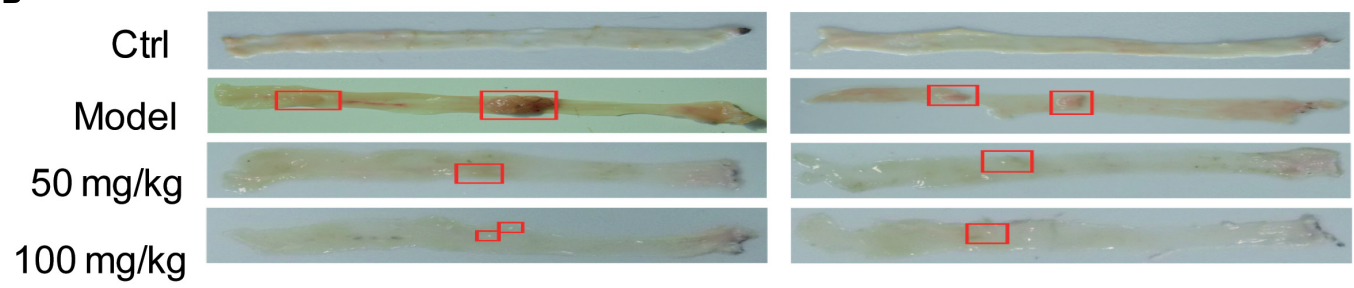

Tumor load

Tumor size percentage
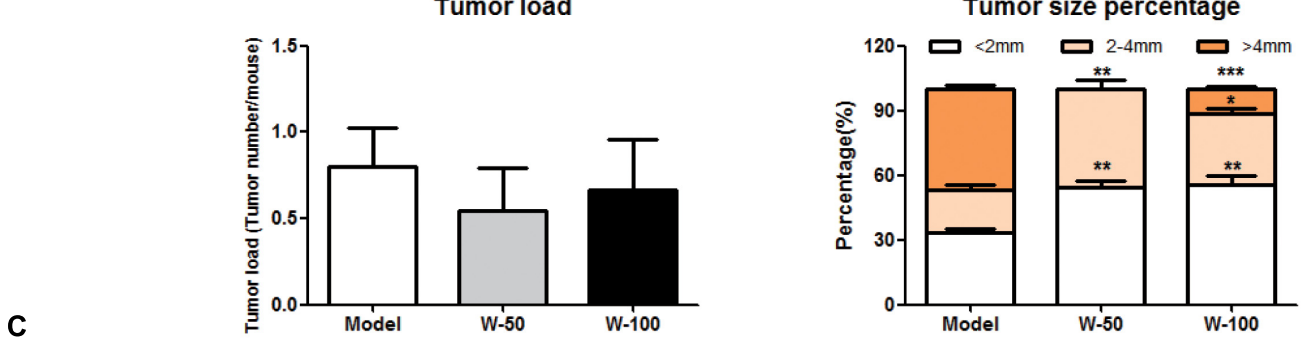

Ctrl
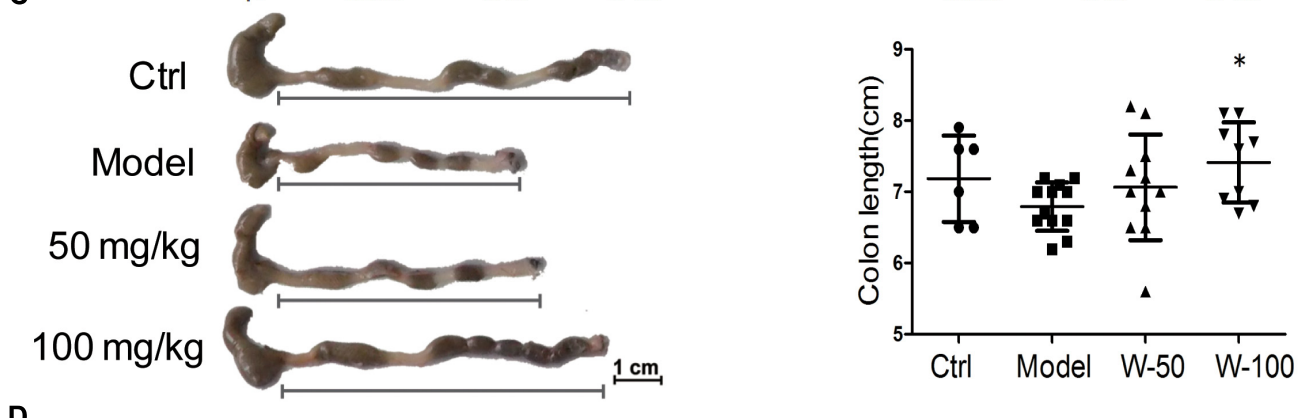

D

\section{Colon cancer tissue}

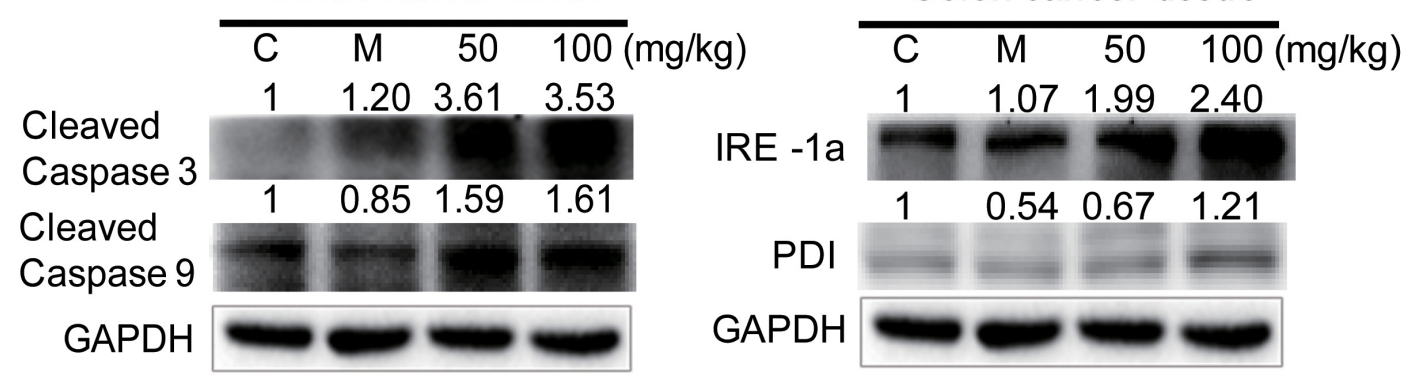

FIGURE 4 | Wogonin inhibited tumor carcinogenesis in AOM/DSS induced CRC. (A) Schematic diagram of AOM/DSS-induced colitis associated colon cancer model ( $n=15$ for each group). (B) The cutaway view of mice colon tissue and the number of tumor load at the endpoint of experiment, tumor size of colonic neoplasms in each group. (C) The colon length changes were measured by using a scaled ruler in each group. (D) Apoptotic and ER-stress related proteins expression after wogonin treatment ( 50 and $100 \mathrm{mg} / \mathrm{kg}$ ) for 21 weeks in mice were determined by western blot. GAPDH was used as an equal loading control, significant difference versus control group, ${ }^{*} P<0.05 ;{ }^{* *} P<0.01 ;{ }^{* * *} P<0.001$.

$100 \mathrm{mg} / \mathrm{kg}$ wogonin treatment group, tumor multiplicity with size of $<2$ and $>4 \mathrm{~mm}$ were 55.55 and $11.11 \%$ in mice treated with $100 \mathrm{mg} / \mathrm{kg}$ wogonin compared with model group 33.34 and $46.67 \%$, respectively (Figure $4 \mathbf{B}$ ). Concurrently, wogonin also preserved colon length to normal from $6.79 \pm 0.34 \mathrm{~cm}$ in model group to $7.06 \pm 0.74$ and 
$7.41 \pm 0.56(P<0.05) \mathrm{cm}$ in wogonin $(50$ and $100 \mathrm{mg} / \mathrm{kg})$ treated groups (Figure 4C).

To determine the effect of wogonin in vivo, western blot was performed to detect apoptotic markers in wogonin treatment groups as compared with model group. Protein expression of cleaved caspase 3 and cleaved caspase 9 were significantly increased by $3.53 \pm 0.13(P<0.001)$ and $1.61 \pm 0.16(P<0.05)$ fold respectively in $100 \mathrm{mg} / \mathrm{kg}$ wogonin treated group. To verify the potential mechanism of wogonin in vivo, ER stress markers were measured in colon tissue of mice with AOM/DSS induced colon cancer. Results showed that, protein level of PDI was increased by $2.34 \pm 0.19(P<0.001)$ fold, and IRE1 $\alpha$ was significantly increased by $2.24 \pm 0.14(P<0.001)$ fold in intestine of mice treated with $100 \mathrm{mg} / \mathrm{kg}$ wogonin as compare with model group (Figure 4D).

\section{DISCUSSION}

Colitis-associated CRC is a chronic inflammation disorder eventually developed into cancer in bowel. Colitis-associated colon cancer is a preventable malignancy, and phytochemicals such as flavonoids (wogonin, curcumin, and quercetin) have been used in CRC chemoprevention in pre-clinical trials by oncologist. Although wogonin has been extensively studied as an anti-tumor flavonoid with acceptable toxicity, not much is known about whether wogonin could prevent colitis associated CRC. In this study, the chemoprevention and chemotherapeutic effects of wogonin were determined and the p53 nucleocytoplamic shuffling involved mechanism was subsequently evaluated. We found that wogonin induces human CRC HCT-116 cell caspasedependent apoptosis through increased ER stress. Subsequently, excessive ER stress inhibited translocation of p53 into the nucleus through increasing phosphor-p53 at S315 and S376 sites, which eventually suppressed and prevented the development of CRC.

Herein, we used AOM-DSS induced CRC mouse model, resemble to human colitis-associated CRC, and found that wogonin exhibited chemopreventive effect in vivo. We found that wogonin successfully prevents CRC tumorigenesis through significantly reducing tumor multiplicity with size of $>4 \mathrm{~mm}$ from 46.67 to $11.11 \%$ (Figure $4 \mathbf{B}, P<0.001$ ). Meanwhile, wogonin reversed colon length to normal from $6.79 \pm 0.34 \mathrm{~cm}$ in AOM/DSS group to $7.41 \pm 0.56 \mathrm{~cm}$ in $100 \mathrm{mg} / \mathrm{kg}$ wogonin treatment group (Figure 4C, $P<0.05$ ). The most important is that wogonin did not show obvious toxicity and there is no significant change in the organ index between mice administrated with wogonin and those with saline (Supplementary Figure 1). These findings indicate wogonin exerts chemopreventive effect without systemic toxicity in colitis-associated CRC.

Next, we explored the underlying mechanism of wogonin exerts chemopreventive effects in vitro. We found that wogonin significantly inhibits the viability and proliferation (Figure 1, $P<0.001)$ in HCT-116 colon cancer cells. Moreover, wogonin specifically induced human CRC HCT-116 cell apoptosis via caspase-dependent pathway (Figure 1D, $P<0.05$ ). What's more, wogonin caused apoptotic cell death through dose-dependently activated ER stress pathway both in vivo and in vitro by increasing ER chaperones (CALNEXIN, PDI), ER-associated key sensors (IRE1 $\alpha$ ), downstream transcription factor $\mathrm{CHOP}$ protein expressions (Figures $\mathbf{2 A}, \mathbf{4}, P<0.05$ ) and targeted genes $D D I 3$ and XBP1 mRNA expressions (Figure 2B, $P<0.001$ ). Previous study revealed that ER stress could directly or indirectly mediate the phosphorylation of p53 and the phosphorylationof p53 at nuclear localization sequence (305-321 bp) sequence blocked the nuclear translocation (Shi and Gu, 2012). In good agreement with previous study, our results further demonstrated that excessive ER stress induced by wogonin ultimately facilitated the cytoplasmic localization of p53 through increasing phosphorp53 at S315 and S376 sites (Figures 3A,C, $P<0.01$ ). Meanwhile, wogonin concentration-dependently reduced the ratio of phosphorylation at serine 15 of p53 in nucleus/cytoplasm (Figure 3B, $P<0.001$ ). Previous study demonstrated that cytoplasmic p53 could inhibit autophagy (Cheong et al., 2012), our study also confirmed that wogonin treatment exhibited the effect of inhibiting autophagy (Figures 1E, 2D, $P<0.05$ ). Potentially, wogonin could have stabilized p53 in the cytoplasm by blockings its export and/or degradation thereby leading to increased levels of total p53 and the localized cytoplasm of p53 induced caspase-dependent apoptosis and inhibited autophagy.

In summary, we found that wogonin could prevent colitisassociated colon cancer in AOM/DSS induced mice model. Multiple pathways were involved in chemopreventive effect of wogonin. These findings provided wogonin as a promising agent for colitis-associated colon cancer chemopreventive agent.

\section{CONCLUSION}

Taken together, by determining regulation p53 nuclear translocation mechanism of wogonin in CRC, our present study advanced our understandings of ER stress directly mediate the phosphorylation of $\mathrm{p} 53$ and the anti-tumor effect of wogonin in following aspects: (i) Subcellular localization of p53 regulates the activity of p53 and affects its function to modulate cellular growth, which implied that regulation of p53 nucleocytoplasmic shuttling offers a novel avenue for the therapeutic strategy in cancer; (ii) Wogonin suppressed CRC tumorigenesis suggested that wogonin is a promising therapeutic candidate for the therapeutic strategy in CRC treatment; (iii) Our mechanistic study uncovered the vital role of wogonin predominantly increased ER stress, which directly mediated the phosphorylation of p53 and ultimately facilitated the cytoplasmic localization of p53, while simultaneously accelerate cellular apoptosis and inhibit autophagy program. The results we confirmed laid a solid pre-clinical foundation for the therapeutic strategy of wogonin in the treatment of CRC through regulating p53 nucleocytoplasmic shuttling.

\section{AUTHOR CONTRIBUTIONS}

LL and ZL conceived the experiments and supervised all research. HW, QF, and JP performed the experiments, analyzed the data, 
and prepared the draft of the manuscript. LL and QF wrote and revised the manuscript. LJ, JH, YW, and XQ provided some technical support. All the authors are accountable for the content of the work.

\section{FUNDING}

This project was supported by the grants of the National Natural Science Foundation of China (Grant Nos. 81473410 and 81503466), the Science and Technology Project of Guangzhou

\section{REFERENCES}

Burada, F., Nicoli, E. R., Ciurea, M. E., Uscatu, D. C., Ioana, M., and Gheonea, D. I. (2015). Autophagy in colorectal cancer: an important switch from physiology to pathology. World J. Gastrointest Oncol. 7, 271-284. doi: 10.4251/wjgo.v7.i11.271

Carrasco, D. R., Sukhdeo, K., Protopopova, M., Sinha, R., Enos, M., Carrasco, D. E., et al. (2007). The differentiation and stress response factor XBP-1 drives multiple myeloma pathogenesis. Cancer Cell 11, 349-360. doi: 10.1016/j.ccr. 2007.02.015

Cheong, H., Lu, C., Lindsten, T., and Thompson, C. B. (2012). Therapeutic targets in cancer cell metabolism and autophagy. Nat. Biotechnol. 30, 671-678. doi: $10.1038 /$ nbt. 2285

Das, V., Kalita, J., and Pal, M. (2016). Predictive and prognostic biomarkers in colorectal cancer: a systematic review of recent advances and challenges. Biomed. Pharmacother. 87, 8. doi: 10.1016/j.biopha.2016.12.064

Fan, Y., Zhang, J., Xiao, W., Lee, K., Li, Z., Wen, J., et al. (2017). Rtnla-mediated endoplasmic reticulum stress in podocyte injury and diabetic nephropathy. Sci. Rep. 7:323. doi: 10.1038/s41598-017-00305-6

Fernandez-Bautista, N., Fernandez-Calvino, L., Munoz, A., and Castellano, M. M. (2017). HOP3, a member of the HOP family in Arabidopsis, interacts with $\mathrm{BiP}$ and plays a major role in the ER stress response. Plant Cell Environ. 40, 1341-1355. doi: 10.1111/pce.12927

Gao, J., Morgan, W. A., Sanchezmedina, A., and Corcoran, O. (2011). The ethanol extract of Scutellaria baicalensis and the active compounds induce cell cycle arrest and apoptosis including upregulation of p53 and Bax in human lung cancer cells. Toxicol. Appl. Pharmacol. 254, 221-228. doi: 10.1016/j.taap.2011. 03.016

Ge, W., Yin, Q., and Xian, H. (2015). Wogonin induced mitochondrial dysfunction and endoplasmic reticulum stress in human malignant neuroblastoma cells via irelalpha-dependent pathway. J. Mol. Neurosci. 56, 652-662. doi: 10.1007/ s12031-015-0530-9

Hu, C. J., Xu, M. Z., Qin, R. J., Chen, W. F., and Xu, X. (2015). Wogonin induces apoptosis and endoplasmic reticulum stress in HL-60 leukemia cells through inhibition of the PI3K-AKT signaling pathway. Oncol. Rep. 33, 3146-3154. doi: 10.3892/or.2015.3896

Iwao, C., and Shidoji, Y. (2014). Induction of nuclear translocation of mutant cytoplasmic p53 by geranylgeranoic acid in a human hepatoma cell line. Sci. Rep. 4:4419. doi: 10.1038/srep04419

Kim, S. J., Kim, H. J., Kim, H. R., Lee, S. H., Cho, S. D., Choi, C. S., et al. (2012). Antitumor actions of baicalein and wogonin in HT-29 human colorectal cancer cells. Mol. Med. Rep. 6:1443. doi: 10.3892/mmr.2012.1085

Lee, D. H., Lee, T. H., Chang, H. J., and Kim, Y. H. (2012). Wogonin induces apoptosis by activating the AMPK and p53 signaling pathways in human glioblastoma cells. Cell. Signal. 24:2216. doi: 10.1016/j.cellsig.2012.07.019

Lee, E., Nichols, P., Spicer, D., Groshen, S., Yu, M. C., and Lee, A. S. (2006). GRP78 as a novel predictor of responsiveness to chemotherapy in breast cancer. Cancer Res. 66, 7849-7853. doi: 10.1158/0008-5472.CAN-06-1660

Levy, J., and Romagnolo, B. (2016). Antitumoral functions of autophagy inhibition in colorectal cancer: the intestinal microbiota and the immune system come to the rescue. Med. Sci. 32, 339-342.

Martínez-Revelles, S., Garcia-Redondo, A. B., Avendaño, M. S., Varona, S., Palao, T., Orriols, M., et al. (2016). Lysyl oxidase induces vascular oxidative stress and contributes to arterial stiffness and abnormal elastin structure in
City (Grant No. 151800014), the Guangdong Natural Science Foundation Province (Grant No. 2015AD030312012), and the Traditional Chinese Medicine Bureau of Guangdong Province (Grant No. 20174004).

\section{SUPPLEMENTARY MATERIAL}

The Supplementary Material for this article can be found online at: https://www.frontiersin.org/articles/10.3389/fphar. 2018.01356/full\#supplementary-material

hypertension: role of p38MAPK. Antioxid. Redox Signal. 27, 379-397. doi: 10.1089/ars.2016.6642

Neska, J., Swoboda, P., Przybyszewska, M., Kotlarz, A., Bolla, N. R., Miłoszewska, J., et al. (2016). The effect of analogues of 1 $\alpha, 25$-dihydroxyvitamin D2 on the regrowth and gene expression of human colon cancer cells refractory to 5fluorouracil. Int. J. Mol. Sci. 17:903. doi: 10.3390/ijms17060903

Ouyang, L., Shi, Z., Zhao, S., Wang, F. T., Zhou, T. T., Liu, B., et al. (2012). Programmed cell death pathways in cancer: a review of apoptosis, autophagy and programmed necrosis. Cell Prolif. 45, 487-498. doi: 10.1111/j.1365-2184. 2012.00845.x

Pyrko, P., Schonthal, A. H., Hofman, F. M., Chen, T. C., and Lee, A. S. (2007). The unfolded protein response regulator GRP78/BiP as a novel target for increasing chemosensitivity in malignant gliomas. Cancer Res. 67, 9809-9816. doi: 10.1158/0008-5472.CAN-07-0625

Qian, C., Wang, Y., Zhong, Y., Tang, J., Zhang, J., Li, Z., et al. (2014). Wogoninenhanced reactive oxygen species-induced apoptosis and potentiated cytotoxic effects of chemotherapeutic agents by suppression Nrf2-mediated signaling in HepG2 cells. Free Radic. Res. 48, 607-621. doi: 10.3109/10715762.2014.89 7342

Qu, L. K., Huang, S., Baltzis, D., Rivas-Estilla, A. M., Pluquet, O., Hatzoglou, M., et al. (2004). Endoplasmic reticulum stress induces p53 cytoplasmic localization and prevents p53-dependent apoptosis by a pathway involving glycogen synthase kinase-3 beta. Genes Dev. 18, 261-277. doi: 10.1101/gad.116 5804

Sakitani, K., Hirata, Y., Hikiba, Y., Hayakawa, Y., Ihara, S., Suzuki, H., et al. (2015). Inhibition of autophagy exerts anti-colon cancer effects via apoptosis induced by 53 activation and ER stress. BMC Cancer 15:795. doi: 10.1186/s12885-0151789-5

Shi, D., and Gu, W. (2012). Dual roles of MDM2 in the regulation of p53: ubiquitination dependent and ubiquitination independent mechanisms of MDM2 repression of p53 activity. Genes Cancer 3, 240-248. doi: 10.1177/ 1947601912455199

Shuda, M., Kondoh, N., Imazeki, N., Tanaka, K., Okada, T., Mori, K., et al. (2003). Activation of the ATF6, XBP1 and grp78 genes in human hepatocellular carcinoma: a possible involvement of the ER stress pathway in hepatocarcinogenesis. J. Hepatol. 38, 605-614. doi: 10.1016/S0168-8278(03) 00029-1

Siegel, R. L., Miller, K. D., Fedewa, S. A., Ahnen, D. J., Meester, R. G. S., Barzi, A., et al. (2017). Colorectal cancer statistics, 2017. CA Cancer J. Clin. 67, 177-193. doi: $10.3322 /$ caac. 21395

Tabas, I., and Ron, D. (2011). Integrating the mechanisms of apoptosis induced by endoplasmic reticulum stress. Nat. Cell Biol. 13, 184-190. doi: 10.1038/ ncb0311-184

Tang, J., Di, J., Cao, H., Bai, J., and Zheng, J. (2015). p53-mediated autophagic regulation: a prospective strategy for cancer therapy. Cancer Lett. 363:101. doi: 10.1016/j.canlet.2015.04.014

Tsai, C. F., Yeh, W. L., Huang, S. M., Tan, T. W., and Lu, D. Y. (2012). Wogonin induces reactive oxygen species production and cell apoptosis in human glioma cancer cells. Int. J. Mol. Sci. 13, 9877-9892. doi: 10.3390/ijms13089877

Xu, S., Zhao, X., Zhao, Q., Zheng, Q., Fang, Z., Yang, X., et al. (2015). Wogonin prevents rat dorsal root ganglion neurons death via inhibiting tunicamycininduced ER stress in vitro. Cell Mol. Neurobiol. 35, 389-398. doi: 10.1007/ s10571-014-0134-x 
Yin, J. J., Li, Y. B., Wang, Y., Liu, G. D., Wang, J., Zhu, X. O., et al. (2012). The role of autophagy in endoplasmic reticulum stress-induced pancreatic beta cell death. Autophagy 8, 158-164. doi: 10.4161/auto.8.2.1 8807

Yu, L., Chen, Y., and Tooze, S. A. (2017). Autophagy pathway: cellular and molecular mechanisms. Autophagy 14, 207-215. doi: 10.1080/15548627.2017. 1378838

Zhang, X., Li, C. F., Zhang, L., Wu, C. Y., Han, L., Jin, G., et al. (2016). TRAF6 restricts p53 mitochondrial translocation, Apoptosis, and tumor suppression. Mol. Cell. 64, 803-814. doi: 10.1016/j.molcel.2016.1 0.002
Conflict of Interest Statement: The authors declare that the research was conducted in the absence of any commercial or financial relationships that could be construed as a potential conflict of interest.

Copyright (c) 2018 Feng, Wang, Pang, Ji, Han, Wang, Qi, Liu and Lu. This is an open-access article distributed under the terms of the Creative Commons Attribution License (CC BY). The use, distribution or reproduction in other forums is permitted, provided the original author(s) and the copyright owner(s) are credited and that the original publication in this journal is cited, in accordance with accepted academic practice. No use, distribution or reproduction is permitted which does not comply with these terms. 